\title{
Método de Custeio Tradicional versus Método da Unidade de Esforço de Produção (UEP): um estudo de caso em uma indústria multiprodutora do segmento metalmecânico
}

\author{
Maíra Vieira da Silva \\ Engenharia de Produção pela UniRitter \\ Rua Orfanatrófio, 555. Bairro Alto-Teresópolis. Porto Alegre/RS. CEP: 90840-440 \\ E-mail: vieiras.maira@gmail.com \\ Ricardo Gonçalves de Faria Corrêa \\ Doutorado em Engenharia de Produção pela Universidade Federal do Rio Grande do \\ Sul - UFRGS \\ Professor na Universidade Federal do Rio Grande - FURG \\ Rua Barão do Cahy, 125. Bairro Cidade Alta. Santo Antônio da Patrulha/RS. CEP: \\ 95500-000 \\ E-mail: ricardofariacorrea@furg.br
}

\section{RESUMO}

Diante do cenário global de competitividade que o mercado apresenta não é surpresa que, para manterem-se na disputa, as organizações precisem alinhar seus processos internos de maneira estratégica. Neste contexto, apesar de nem sempre receber a devida importância, um sistema de custeio aprimorado e voltado à tomada de decisão gerencial, através de seus princípios e métodos, apresenta-se como um diferencial. As metodologias de custeio tradicionalmente adotadas tendem a não atender satisfatoriamente as necessidades das empresas nesta esfera, principalmente as multiprodutoras. Tem-se, a partir de então, como objetivo avaliar o resultado da aplicação do método da UEP frente à utilização do método dos CC, a fim de propor um método de custeio mais apropriado ao processo de transformação de uma indústria multiprodutora do segmento metalmecânico. O estudo é limitado ao processo de usinagem da empresa, utilizando-se da aplicação de um projeto-piloto, sendo conduzido pela metodologia de estudo de caso. Como resultado apresentam-se a aplicação do método da Unidade de Esforço de Produção (UEP) e a análise comparativa entre os resultados obtidos por tal aplicação versus os resultados apresentados pelo método tradicional dos Centros de Custos (CC). Por fim, verifica-se que o método utilizado atualmente pela empresa - método dos CC - não apresenta condições de acurácia quanto ao custeio final unitário dos produtos fabricados pela empresa. No entanto, o método da UEP apresenta-se como uma alternativa interessante para o caso, abrindo campo para pesquisas futuras.

Palavras chave: Sistemas de Custeio. Métodos de Custeio. Método da Unidade de Esforço de Produção (UEP). Tomada de Decisão Gerencial. 
Método de Custeio Tradicional versus Método da Unidade de Esforço de Produção (UEP): um estudo de caso em uma indústria multiprodutora do segmento metalmecânico Maíra Vieira da Silva, Ricardo Gonçalves de Faria Corrêa

\title{
Traditional Costing Method versus Production effort unit (UEP) Method: a case study in a multi-producer industry in the metal-mechanical segment
}

\begin{abstract}
Given the global competitiveness scenario in the market, it is not surprising that, in order to be able to dispute, organizations need to strategically align their internal processes. In this context, although not always receiving due importance, an improved costing system and focused on managerial decision making, through its principles and methods, presents itself as a differential. The traditionally adopted costing methodologies tend not to satisfactorily meet the needs of companies in this sphere, especially multi-producers ones. The objective of this study is to evaluate the result of applying the Production Effort Unit (UEP) method by comparing it to the use of the Cost Centers (CC) method, in order to propose a more appropriate costing method to the transformation process of a multi-producer industry in the metal-mechanical segment. The study is limited to the company's machining process, using the application of a pilot project, being conducted by the case study methodology. As a result, the application of the Production Effort Unit (UEP) method is presented and the comparative analysis between the results obtained. It appears to be that the method currently used by the company - CC method - does not present conditions of accuracy regarding the final unit cost of the products manufactured by the company. However, the UEP method presents itself as an interesting alternative for the case, opening the field for future research.
\end{abstract}

Key-words: Costing Systems. Costing Methods. Production Effort Unit (UEP) method. Management Decision Making.

\section{Método de Costeo Tradicional versus Método de la Unidad de Esfuerzo de Producción (UEP): un estudio de caso en una industria multiproducto del sector metalmecánico}

\section{RESUMEN}

Frente al escenario global de competitividad que el mercado presenta, no es sorprendente que para mantenerse en la disputa, las organizaciones necesitan alinear sus procesos internos de forma estratégica. En este contexto, a pesar de no siempre recibir la debida importancia, un sistema de costeo mejorado y enfocado en la toma de decisión gerencial, a través de sus principios y métodos, se presenta como un diferencial. Las metodologías de costeo tradicionalmente adoptadas tienden a no atender satisfactoriamente las necesidades de las empresas en este ámbito, principalmente las empresas multiproducto. A partir de entonces, el objetivo es evaluar el resultado de la aplicación del método de Unidad de Esfuerzo de Producción (UEP) frente al uso del método de Centros de Costos (CC) con el fin de proponer un método 
Método de Custeio Tradicional versus Método da Unidade de Esforço de Produção (UEP): um estudo de caso em uma indústria multiprodutora do segmento metalmecânico Maíra Vieira da Silva, Ricardo Gonçalves de Faria Corrêa

de costeo más adecuado al proceso de transformación de una industria multiproducto del segmento metal-mecánico. El estudio se limita al proceso de mecanizado de la empresa, mediante la aplicación de un proyecto piloto, siendo realizado por la metodología de estudio de caso. Como resultado se presentan las etapas de aplicación del método UEP y el análisis comparativo entre los resultados obtenidos por dicha aplicación versus los resultados presentados por el método tradicional de CC. Finalmente se verifica que el método utilizado actualmente por la empresa - método CC - no presenta un servicio satisfactorio en cuanto al costo unitario final de los productos fabricados, sin embargo, el método UEP se presenta como una alternativa interesante para el caso, abriendo campo para estudios futuros.

Palabras clave: Sistemas de Costeo. Métodos de Costeo. Método de Unidad de Esfuerzo de Producción (UEP). Toma de Decisión Gerencial.

\section{INTRODUÇÃO}

Atualmente é de comum conhecimento que o cenário do mercado de maneira global está a cada dia mais competitivo. Diante disso, Bornia (2010) diz que as organizações que não tiverem competência de mensurar a performance de seus processos, para então intervir de maneira ágil e corrigi-los, estarão ameaçadas diante de uma concorrência com maior eficiência. Nesse contexto, apesar de normalmente não receber devida atenção dentro das organizações, o enfoque no sistema de custeio adotado pode se mostrar como um forte aliado.

Dito isso, visando contribuir para e através de um maior proveito do sistema de custeio, o tema deste trabalho concentra-se em um de seus pilares - o método de custeio. Para tanto, contrastou-se a adoção de uma metodologia de custeio tradicional, o método dos Centros de Custos (CC), versus o método da Unidade de Esforço de Produção (UEP), usando para isso a perspectiva de estudo de caso. Segundo Silva, Cruz, Santos e Leone (2018), a comparação de métodos de custeio e a mudança de método adotado podem melhorar a eficácia da gestão estratégica de custos.

Apesar da abordagem relacionada às ofertas dos métodos de custeio não ser uma novidade (Antunes Junior, 1988; Kliemann Neto, 1994; Kraemer, 1995; Muller, 1996), a crença em estudar e comparar métodos com o intuito de uma utilização mais 
Método de Custeio Tradicional versus Método da Unidade de Esforço de Produção (UEP): um estudo de caso em uma indústria multiprodutora do segmento metalmecânico Maíra Vieira da Silva, Ricardo Gonçalves de Faria Corrêa

apropriada nasce do fato de que, na prática, muitas empresas ainda não desempenham esse comportamento (Villarmois \& Levant, 2011). Ainda, não se questionam quanto a essa utilização e não a exploram em seu favor. Esse também é o caso da empresa inserida neste estudo.

A problemática reside no fato de que a empresa produz distintos produtos com tecnologias e esforços diferenciados. Contudo, apesar das diferenciações demandadas por cada produto, esses compartilham os recursos durante o processo produtivo, com isso tem-se início a dificuldade em relação à apuração dos custos. A unidade de negócio abordada neste trabalho possui um mix de produção com um total de 76 produtos, os quais apresentam custos unitários significativamente diferentes do custo médio unitário do mix de produção, assegurando o quão arriscado e inadequado é o fato de se basear em apurações de custos generalistas. A alta heterogeneidade da produção e o extenso mix de produção combinados com as recomendações de Valentim (2018) tornam o método da UEP adequado a essa realidade. Além disso, o método é recomendado como fonte geradora de informações gerenciais (Guimarães Filho, Bristot, Marques, Feil, \& Colombo, 2016; Zanin, Bilibio, Pacassa, \& Cambruzzi, 2019) e por proporcionar uma relação custo-benefício da informação positiva (Lembeck \& Wernke, 2019).

Frente a essa problemática, o trabalho visa contribuir de maneira a responder a seguinte questão: A metodologia de custeio de produção atual representa em custos o que acontece na prática dos processos de produção? Para tanto, o objetivo deste estudo está em avaliar o resultado da aplicação do método da UEP frente à utilização do método dos CC, a fim de propor um método de custeio mais apropriado ao processo de transformação de uma indústria multiprodutora do segmento metalmecânico.

A melhor adequação do método de custeio tem por finalidade subsidiar o processo decisório. Dessa forma, este trabalho contribui também para suprir as necessidades de pesquisa apontadas por Pasa, Nascimento e Correio (2017). 
Método de Custeio Tradicional versus Método da Unidade de Esforço de Produção (UEP): um estudo de caso em uma indústria multiprodutora do segmento metalmecânico Maíra Vieira da Silva, Ricardo Gonçalves de Faria Corrêa

\section{REFERENCIAL TEÓRICO}

As funções de um sistema de custeio, conforme relatam Bornia (2010) e Crepaldi \& Crepaldi (2017), são avaliação de estoques, auxílio ao controle e apoio a tomada de decisão gerencial. De acordo com Souza \& Clemente (2011) os sistemas de custeio, apesar da evolução que as empresas tiveram em seus processos produtivos, seguem com o escopo tradicional com o qual surgiram. Bornia (2010) e Padoveze (2010) acrescentam que com a evolução da tecnologia e dos sistemas de informação, o custo para a operacionalização de sistemas de custeio não é mais um problema.

Os sistemas de custeio são constituídos pela combinação entre princípios de custeio e métodos de custeio, que respectivamente têm por característica responder que custo será considerado e como será considerado (Souza \& Diehl, 2009; Bornia, 2010). O princípio de custeio norteia a informação que se deseja obter através do sistema de custeio, enquanto o método de custeio tem por finalidade dar vida a esta informação, viabilizando sua operacionalização (Fadanelli, 2007; Bornia, 2010).

Fadanelli (2007) relata que o método de custeio serve de base para a tomada de decisão. Oliveira \& Allora (2010) descrevem que o saber preciso em relação ao custo de cada um de seus produtos oferece à empresa a possibilidade de escolha por um mix de produção mais rentável.

Diversos são os métodos de custeio existentes. Os métodos de Custo Padrão (CP) e Centros de Custos (CC) são descritos como metodologias tradicionais de custeio, enquanto os métodos Custeio Baseado em Atividades (ABC), Unidade de Esforço de Produção (UEP) e Custeio Baseado em Atividade e Tempo (TDABC) são considerados métodos contemporâneos, modernos ou estratégicos (Kraemer, 1995; Muller, 1996; Oliveira \& Allora, 2010). Nas subseções seguintes são apresentados os métodos do CC e UEP. 
Método de Custeio Tradicional versus Método da Unidade de Esforço de Produção (UEP): um estudo de caso em uma indústria multiprodutora do segmento metalmecânico Maíra Vieira da Silva, Ricardo Gonçalves de Faria Corrêa

\subsection{Método dos Centros de Custos (CC)}

De acordo com Fadanelli (2007) e Campagnolo, Souza e Kliemann Neto (2009), a sistemática do método dos CC representa os procedimentos da contabilidade tradicional de custos, sendo por esse motivo o método de custeio mais utilizado no Brasil. A principal característica do método dos CC, segundo Bornia (2010), é a divisão da empresa em centros de custo, para que posterior a isso, através de bases de distribuição, os custos possam ser repassados aos produtos. Segundo Muller (1996), os centros de custos podem ser classificados em:

i) centros comuns: têm a função de prestar serviços para todos os demais centros (ex.: diretoria, departamento jurídico e recursos humanos);

ii) centros auxiliares: têm a função de fornecer serviços que suportem a operação dos centros produtivos, (ex.: áreas de engenharia, manutenção, planejamento e controle da produção);

iii) centros produtivos: são os centros onde o processo de transformação do produto ou serviço ocorre.

Os centros comuns e auxiliares possuem relação indireta com os produtos, enquanto os centros produtivos apresentam relação direta com os mesmos. No entanto, os centros produtivos utilizam dos serviços prestados pelos centros indiretos, e por esse motivo, além de seus custos diretos, recebem também uma parcela dos demais centros de custos indiretos, através de critérios de rateio (Fadanelli, 2007). Segundo Bornia (2010), as etapas do método dos CC constituem-se em separar os custos em itens, agrupando-os por suas naturezas distintas, dividir a empresa em centros de custos, de modo a manter a homogeneidade em cada centro, realizar a identificação dos custos com os centros por meio da distribuição primária, realizar o direcionamento dos custos de centros indiretos até os centros produtivos, por intermédio da distribuição secundária e por fim realizar a distribuição final dos custos dos centros produtivos até os produtos.

De acordo com Kraemer (1995), o método dos CC é adequado quando os processos apresentam poucas diferenciações entre si, tendo em sua maioria equipamentos iguais ou similares, com um operador destinado ao atendimento de uma 
Método de Custeio Tradicional versus Método da Unidade de Esforço de Produção (UEP): um estudo de caso em uma indústria multiprodutora do segmento metalmecânico Maíra Vieira da Silva, Ricardo Gonçalves de Faria Corrêa

única máquina. No entanto, diante do cenário de evolução tecnológica, aumento da variedade de produtos, heterogeneidade e complexidade dos processos fabris, o método esbarro na limitação de seus pressupostos.

\subsection{Método da Unidade de Esforço de Produção (UEP)}

De acordo com Allora e Allora (1995), todas as informações passam a ter relevância somente quando são atreladas aos itens que foram produzidos em determinado período. No entanto, em empresas multiprodutoras, principalmente em indústrias de transformação, a sistemática de direcionamento dos custos até os produtos pode ser bastante complexa, bem como a gestão desses custos, visto que não é apenas um único produto que os gera, mas sim um composto de distintos produtos (Valentim, 2018).

O método da UEP visa apropriar e monitorar custos nesse contexto de produção diversificada. A partir disso, visa possibilitar a unificação da produção em uma medida única (UEP) e equivalente para todo o processo produtivo, apresentando-se como base de comparação direta entre os produtos e processos produtivos (Fadanelli, 2007; Bornia, 2010). De acordo com Souza \& Diehl (2009) essa medida é determinada através de uma sistemática que ao final permite a obtenção de um valor de referência, que é utilizado para a comparação dos produtos, quaisquer que sejam. Conforme Kliemann Neto (1994), a unidade abordada pelo método da UEP baseia-se na quantificação da noção do esforço de produção que ocorre no processo de transformar a MP em produto acabado. Tal esforço é representado pela soma de todos os esforços exercidos em cada uma das operações da fábrica.

Kliemann Neto (1994), Muller (1996) e Oliveira e Allora (2010) descrevem três princípios que fundamentam a sistemática do método da UEP, sendo eles: Princípio do valor agregado, Princípio das relações constantes e Princípio das estratificações. $O$ Princípio do valor agregado, de acordo com Kliemann Neto (1994) e Muller (1996), considera que o produto de uma indústria é, na verdade, o trabalho realizado sobre a matéria prima (MP) para transformá-la em produto acabado. Já o Princípio das relações 
Método de Custeio Tradicional versus Método da Unidade de Esforço de Produção (UEP): um estudo de caso em uma indústria multiprodutora do segmento metalmecânico Maíra Vieira da Silva, Ricardo Gonçalves de Faria Corrêa

constantes, de acordo com Muller (1996), assegura que independente das variações econômicas que ocorram, as relações de esforços entre os Postos Operativos (PO) se mantêm constantes no tempo. E o Princípio das estratificações está intimamente relacionado com o princípio anterior, pois conforme Fernandes (2003) e Oliveira \& Allora (2010) o grau de diferenciação na estratificação dos itens de custos, entendidos como as contas a serem consideradas, impactam diretamente nos resultados. Isto, de acordo com o princípio das estratificações, quer dizer que deve haver comprometimento com o direcionamento dos itens de custos aos POs (Fernandes, 2003), evitando-se o uso de arbitrariedade para tal ou até mesmo que se deixe de considerar esses itens como imputáveis diretamente aos POs.

A sistemática de funcionamento, no que tange as etapas de aplicação do método da UEP, será apresentada juntamente com a seção de resultados deste trabalho, acompanhando as etapas de aplicação do método na empresa onde ocorreu o estudo de caso.

\section{METODOLOGIA}

Nessa seção é descrita a classificação da pesquisa, o cenário da empresa de estudo e o método de trabalho utilizado.

\subsection{Classificação da Pesquisa}

Quanto à sua finalidade este estudo classifica-se como pesquisa aplicada, tendo por característica a função de contribuir através da avaliação e (possível) solução de problemas existentes no meio em que o pesquisador está inserido (Gil, 2018). Do ponto de vista da abordagem do problema, esta pesquisa classifica-se como qualitativa, pois utilizou de passos como entrevistas despadronizadas e visitas para o entendimento da problemática estudada (Creswell, 2010). Além disso, assume também caráter qualitativo quando os dados são analisados na etapa final de análise de resultados (Martins, 2004). O método adotado para a condução da pesquisa foi o de estudo de 
Método de Custeio Tradicional versus Método da Unidade de Esforço de Produção (UEP): um estudo de caso em uma indústria multiprodutora do segmento metalmecânico Maíra Vieira da Silva, Ricardo Gonçalves de Faria Corrêa

caso, que conforme descrito por Dresch, Lacerda e Miguel (2015) "[...] quando bem conduzido, proporciona uma compreensão de determinados fenômenos em profundidade, além de ser bastante comum para estudos empíricos."

\subsection{A Empresa e seu Cenário}

A organização onde se destinou a aplicação deste trabalho é uma indústria multinacional consolidada no segmento metalmecânico. O grupo conta com nove plantas produtivas e o estudo desenvolvido está limitado à planta situada no Brasil, na região do Vale dos Sinos (RS). Essa planta divide-se ainda em duas unidades de negócio (unidades 1 e 2 ) e o sistema de custeio adotado é comum a ambas. Ao considerar o amplo número de processos e sua complexidade, optou-se pela definição de um único processo para ser analisado, como forma de projeto-piloto.

A empresa abordada fabrica produtos para o mercado de exportação e doméstico. Entre o volume de produtos fabricados, estimado em 8.000 .000 de peças no ano de 2019 , em torno de $90 \%$, destinam-se a atender os mercados norte-americano, alemão e chinês. Os clientes externos são as demais unidades de produção do grupo, sendo a unidade do Brasil responsável por fornecer em torno de $80 \%$ desse tipo de produto para o grupo, ocupando assim um papel importante. Os outros $10 \%$ do volume fabricado são destinados a atender o mercado interno brasileiro.

São três linhas de produtos fabricados: profissional, intermediário e hobby. A linha profissional é um produto mais robusto, possuindo para tal, requisitos técnicos diferenciados. Já a linha hobby destina-se ao uso ocasional (residencial), não sendo um produto que realiza tanto esforço em seu uso pelo cliente final. O intermediário, por sua vez, mostra-se como uma opção entre os dois extremos.

Cada linha de produto possui uma complexidade e diferenciação em seu processo de fabricação. Com o intuito de trazer um entendimento em relação à diferenciação dos produtos a serem abordados no estudo de caso, a Figura 1 apresenta a sistemática de nomenclatura dos produtos. 
Método de Custeio Tradicional versus Método da Unidade de Esforço de Produção (UEP): um estudo de caso em uma indústria multiprodutora do segmento metalmecânico Maíra Vieira da Silva, Ricardo Gonçalves de Faria Corrêa

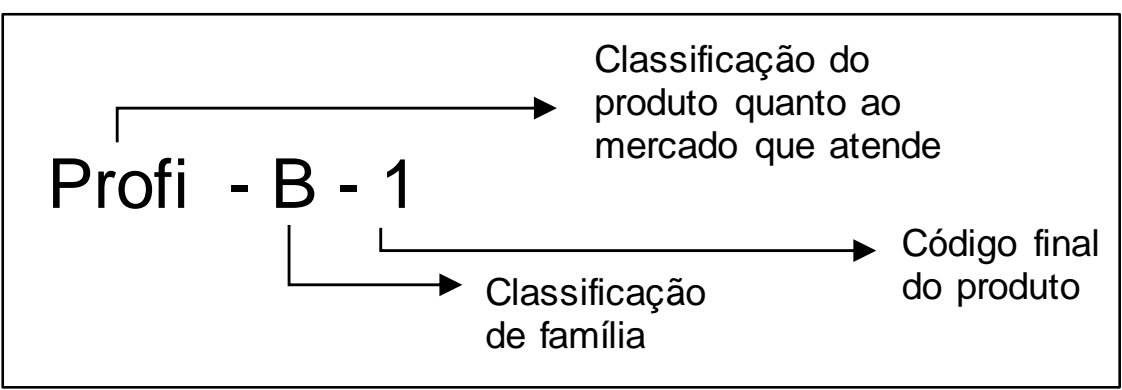

Figura 1. Sistemática de nomenclatura dos produtos

Fonte: Dados as pesquisa.

Existem seis famílias de produtos conforme descrição contida no Quadro 1. Suas diferenças serão retomadas na análise de resultados deste estudo.

\begin{tabular}{|c|c|}
\hline Família & Descrição Família \\
\hline A & Produtos Fundidos por Gravidade \\
\hline B & Produto de Tecnologia diferenciadadesenvolvida pela empresa \\
\hline C & Produtos Fundidos sob pressão \\
\hline D & Produtos com bucha \\
\hline E & Produtos com tampa colada \\
\hline F & Produtos com tampa parafusada \\
\hline
\end{tabular}

Quadro 1. Descrição das famílias de produtos

Fonte: Dados da pesquisa.

Os dados apresentados na seção de resultados foram multiplicados por uma constante para fins de confidencialidade, sendo as relações de proporcionalidade mantidas. O cenário abordado no estudo corresponde ao ano de 2019.

\subsection{Método de Trabalho, Ferramentas e Técnicas}

A Figura 2 exibe o fluxo do método de trabalho. Suas etapas são detalhadas nos parágrafos seguintes. 
Método de Custeio Tradicional versus Método da Unidade de Esforço de Produção (UEP): um estudo de caso em uma indústria multiprodutora do segmento metalmecânico Maíra Vieira da Silva, Ricardo Gonçalves de Faria Corrêa

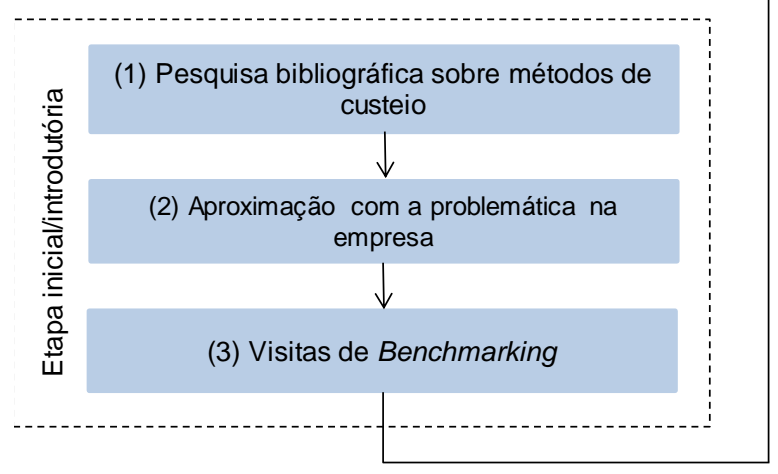

Etapas contempladas no capítulo de resultados .

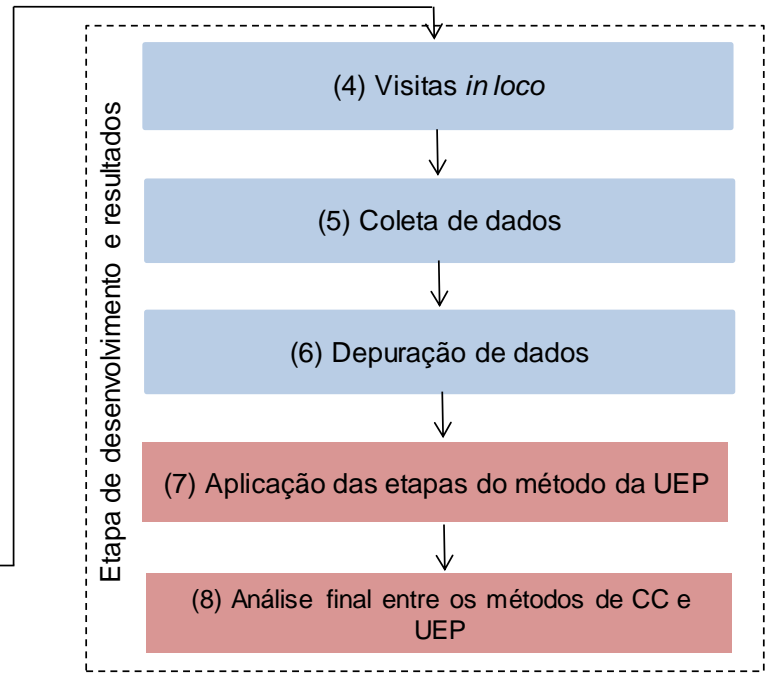

Figura 2. Método de trabalho

Fonte: Dados da pesquisa.

$\mathrm{Na}$ primeira etapa buscou-se o conhecimento do que a literatura oferece em relação a métodos de custeio. A busca deu-se especialmente em livros voltados ao ensinamento de custos na Engenharia de Produção.

A segunda etapa ocorreu através de entrevistas semiestruturadas com gerentes de produção, especialista de custos, supervisores de produção e troca rápida de ferramentas (TRF), analista de processos e analista de planejamento. A condução das entrevistas se apoiou em tópicos predefinidos pelos pesquisadores, conforme descrito por Marconi \& Lakatos (2017).

$\mathrm{Na}$ terceira etapa, buscou-se o conhecimento de empresas que fazem ou fizeram uso do método da UEP para o custeio da produção, com o intuito de realizar visitas de benchmarking. Foram encontradas duas empresas na região, as quais viabilizaram a visita para troca de conhecimentos.

$\mathrm{Na}$ quarta etapa ocorreram as visitas in loco ao processo de usinagem, com o objetivo de realizar observações acerca do processo de transformação que ali ocorre, promovendo maior entendimento, aproximação e avaliação para as fases seguintes.

$\mathrm{Na}$ quinta etapa deu-se início a coleta de dados, através da geração de relatórios, via sistema utilizado pela empresa e através do acesso a planilhas 
Método de Custeio Tradicional versus Método da Unidade de Esforço de Produção (UEP): um estudo de caso em uma indústria multiprodutora do segmento metalmecânico Maíra Vieira da Silva, Ricardo Gonçalves de Faria Corrêa

eletrônicas utilizadas para fins de controle. Os dados que aqui foram coletados referemse a custos relacionados ao processo de usinagem e a tempos de roteiro dos produtos neste processo.

A etapa de depuração dos dados teve como objetivo verificar possíveis faltas de registros ou até mesmo incoerência nos registros, a fim de garantir seu uso de maneira adequada, de modo a não comprometer o resultado do estudo. Foram realizadas conversas de feedback com os responsáveis pela geração dos dados em sistema e/ou planilhas, validando ou não seu uso na sequência do estudo de caso.

Posteriormente, deu-se início às etapas de aplicação do método da UEP, conforme exposto na seção de resultados. E, por fim, após às etapas de aplicação do método da UEP, analisou-se comparativamente os métodos CC e UEP, apresentando o custo unitário dos produtos e discutindo o impacto nas decisões da empresa.

\section{RESULTADOS}

Nessa seção é apresentado o processo de custeio na empresa, seguido das etapas de aplicação do método da UEP e seus resultados.

\subsection{Elementos de Composição de Custo dos Produtos}

O produto é composto cinco elementos de custo:

i) Custo Máquina: custos incorridos com a depreciação de máquinas e equipamentos, manutenção, materiais diversos, alocação de utilidades (água, energia elétrica e tratamento de efluentes), área ocupada e alocação de mão de obra indireta (MOI);

ii) Material: custo de matéria-prima;

iii) MOD: custo de MOD incorrido de acordo com o roteiro do produto no processo produtivo, sendo representado pelo salário, benefícios e obrigações vinculados a colaboradores diretamente relacionados à produção; 
Método de Custeio Tradicional versus Método da Unidade de Esforço de Produção (UEP): um estudo de caso em uma indústria multiprodutora do segmento metalmecânico

Maíra Vieira da Silva, Ricardo Gonçalves de Faria Corrêa

iv) Dispositivos e Moldes: custos incorridos com a depreciação e manutenção de dispositivos e moldes para a fabricação dos produtos;

v) Logística: custos das áreas de planejamento, compras e gerenciamento de estoques, bem como os custos da movimentação interna de materiais.

No processo de usinagem, os elementos de custos envolvidos são somente custo máquina, MOD e dispositivos e moldes. Dado que os demais elementos constituem o produto como um todo, não sendo separados por processos de fabricação. A aplicação deste trabalho ocorre no elemento de custo máquina por representar 73\% (R\$ 9.400.183,68) dos custos de usinagem (ver Tabela 1 detalhada por contas), considerando volume e mix de produção planejados.

Tabela 1

Custo total CC usinagem

\begin{tabular}{lrr}
\hline Descrição conta & \multicolumn{2}{c}{ Valor } \\
\hline Manutenção & $\mathrm{R} \$$ & $1.478 .126,71$ \\
Lubrificantes & $\mathrm{R} \$$ & $414.502,67$ \\
Produtos Químicos & $\mathrm{R} \$$ & $35.802,11$ \\
Materiais Diversos & $\mathrm{R} \$$ & $108.305,99$ \\
Material para embalagem & $\mathrm{R} \$$ & 469,84 \\
Ferramentas & $\mathrm{R} \$$ & $1.735 .950,56$ \\
Depreciação & $\mathrm{R} \$$ & $2.178 .063,41$ \\
Tempo adicional e perdas & $\mathrm{R} \$$ & $556.576,35$ \\
Área & $\mathrm{R} \$$ & $249.265,76$ \\
Ar comprimido & $\mathrm{R} \$$ & $149.541,69$ \\
Energia & $\mathrm{R} \$$ & $941.752,38$ \\
Água & $\mathrm{R} \$$ & $63.797,57$ \\
Tratamento de efluentes & $\mathrm{R} \$$ & $9.897,76$ \\
Alocações MOI & $\mathrm{R} \$$ & $1.478 .130,88$ \\
\hline Custo Total & $\mathrm{R} \$$ & $\mathbf{9 . 4 0 0 . 1 8 3 , 6 8}$ \\
\hline
\end{tabular}

Nota. Fonte: Dados da pesquisa. 
Método de Custeio Tradicional versus Método da Unidade de Esforço de Produção (UEP): um estudo de caso em uma indústria multiprodutora do segmento metalmecânico Maíra Vieira da Silva, Ricardo Gonçalves de Faria Corrêa

\subsection{Método de Custeio Atual}

Os custos indiretos são direcionados até o produto através do elemento de custo máquina. O CC usinagem contempla um total de 49 máquinas, que possuem diferenciações entre si. O funcionamento dessa estrutura, de acordo com o nível de atividade de produção e com o custo fixo atrelado, gera um custo total absoluto que é direcionado ao produto através de uma tarifa hora $(\mathrm{R} \$)$. A Tabela 1 apresenta as contas que compõem o valor absoluto para esse CC. Os valores informados já consideram as distribuições primárias e secundárias de custos, de acordo com as bases e critérios de rateio utilizados pela empresa.

O CC usinagem possui um planejamento de 268.456 horas de produção para o ano de 2019, sendo assim, através da divisão de custo total pelo número de horas planejadas tem-se a tarifa hora de $\mathrm{R} \$ 35,02$. Exemplificando: se um produto tem $\mathrm{o}$ tempo de usinagem de 0,12 horas então seu custo no processo de usinagem será de $R \$ 4,20(R \$ 35,02 \times 0,12 h)$.

A única variável que exerce diferenciação no custeio final dos produtos é o tempo. Assim, o método pressupõe que os recursos do CC são homogêneos, enquanto na realidade não o são, pois há diferença de máquinas, níveis de tecnologia, demanda por manutenção, etc. Dessa forma, independe do recurso que o produto demanda no processo, seu custo unitário está atrelado somente ao tempo. Sendo assim, um produto que em seu roteiro de produção utiliza de um recurso mais caro, porém com tempo de processamento menor, será custeado com um valor inferior a um produto que utiliza de um recurso mais barato, porém mais lento.

\subsection{Aplicação do Método da UEP}

A aplicação do método da UEP se deu em cinco etapas. Optou-se por considerar nesta aplicação apenas as contas que possuem apontamentos e dados detalhados e rastreáveis. Desse modo, consideraram-se as contas de depreciação, manutenção e energia. Considerou-se $77,48 \%$ do valor da conta de depreciação (que corresponde ao valor de $\mathrm{R} \$ 1.687 .642,43)$, pois este é o percentual de dados com rastreabilidade. As 
Método de Custeio Tradicional versus Método da Unidade de Esforço de Produção (UEP): um estudo de caso em uma indústria multiprodutora do segmento metalmecânico Maíra Vieira da Silva, Ricardo Gonçalves de Faria Corrêa

demais contas em que se utilizou no método da UEP, utilizou-se $100 \%$ do seu valor, totalizando assim $R \$ 4.107 .521,51$. Logo, ao voltar para a Tabela 1, sabe-se que o valor contemplado nessa aplicação corresponde ao percentual de $43,70 \%$ do valor total do CC usinagem.

A utilização de apenas três contas ocorre à luz do Princípio das estratificações, a fim de garantir precisão nos resultados. Assim, deu-se preferência por não considerar as contas que não possuem condições de direcionamento exato aos POs, ou então, de considerá-las por intermédio de arbitrariedade.

\subsubsection{Divisão dos Postos Operativos (POs)}

A divisão dos POs se deu através de observações diretas e entendimento acerca do processo produtivo, por intermédio da etapa de visitas in loco ao processo de usinagem. O processo foi dividido em quatro POs (Tabela 2). A divisão de POs se deu com o intuito de manter recursos homogêneos.

Tabela 2

\section{Divisão dos POs}

\begin{tabular}{lcccccc}
\hline & PO1 & PO2 & PO3 & PO4 & \multicolumn{1}{l}{ Total } \\
\hline Número total de máquinas & 40 & 3 & & 4 & 2 & \multicolumn{2}{c}{49} \\
Horas planejadas 2019 & 219824 & & 14.115 & 20.945 & 13.573 & 268.456 \\
\hline
\end{tabular}

Nota. Fonte: Dados da pesquisa.

O PO1 contempla um total de 40 máquinas Chirons FZ (Spindle único) com tempo de utilização desde a data de aquisição entre 7 e 15 anos, estando em sua maioria na segunda fase de depreciação. Para compreensão, a empresa trabalha com o modelo de depreciação linear, tendo como regra que o equipamento seja totalmente depreciado em 7 anos de vida, a partir de então, o equipamento passa a ter seu custo de depreciação advindo do que é denominado como segunda fase de depreciação, isto é, tem o valor de $50 \%$ de seu custo de aquisição novamente considerado para uma 
Método de Custeio Tradicional versus Método da Unidade de Esforço de Produção (UEP): um estudo de caso em uma indústria multiprodutora do segmento metalmecânico Maíra Vieira da Silva, Ricardo Gonçalves de Faria Corrêa

depreciação em 7 anos. Além disso, as máquinas contempladas contam com um nível baixo de tecnologia se comparado às demais.

O PO2 contempla um total de 3 máquinas também Chirons FZ (Spindle único) com tempo médio de vida de 12,6 anos, estando essas, igualmente ao PO1 na segunda fase de depreciação. Todavia, esse grupo de máquinas possui uma célula automatizada para alimentação durante o processo produtivo.

O PO3 contempla um total de 4 máquinas Chirons DZ (Spindle duplo), essas máquinas se comparadas as máquinas do PO1 e PO2, possuem uma capacidade dupla de usinagem, uma vez que possuem um Spindle a mais. Assim, são máquinas com valor de aquisição alto, além disso, possuem em média 3,5 anos de vida atualmente, estando assim em sua primeira fase de depreciação.

O PO4 possui um total de 2 máquinas Chirons FZ (Spindle único), porém 100\% automatizada. Essas máquinas estão em sua primeira fase de depreciação.

\subsubsection{Cálculo dos Foto-Índices dos POs (FIPOs)}

A etapa de cálculo dos FIPOs constitui-se em direcionar aos POs cada uma das contas definidas, para então chegar-se ao custo hora de cada um dos POs. A conta de depreciação foi direcionada de acordo com cada uma das máquinas que se encontra em cada um dos POs, a partir da identificação em relatório de ativos da empresa. A conta de manutenção foi aberta em materiais, horas e serviços, que respectivamente correspondem aos gastos com os materiais utilizados para realizar a manutenção dos equipamentos, às alocações de $\mathrm{MOI}$ para a realização das manutenções, e às horas de manutenção destinadas a cada máquina.

A conta de energia elétrica, por sua vez, foi direcionada aos POs através de critério subjetivo apoiado em análise de especialistas. Nesse caso, através de uma matriz de pontuação, especialistas de usinagem e de automação atribuíram pesos distintos para cada uma das famílias de máquinas. Foram utilizados três tipos de pesos para consumo de energia elétrica: econômico $(0,9)$, padrão $(1)$, e alto consumo $(1,5)$. Os POs são compostos por máquinas com pesos distintos, resultando em uma 
Método de Custeio Tradicional versus Método da Unidade de Esforço de Produção (UEP): um estudo de caso em uma indústria multiprodutora do segmento metalmecânico Maíra Vieira da Silva, Ricardo Gonçalves de Faria Corrêa

pontuação total que foi utilizada para direcionar o custo de energia elétrica, proporcionalmente a pontuação somada em cada PO sobre o valor total da conta de energia elétrica. A Tabela 3 apresenta parte da sistemática.

Tabela 3

Pontuação em relação ao consumo de energia elétrica por família de máquinas

\begin{tabular}{|c|c|c|c|c|c|c|}
\hline Descrição da família & $\begin{array}{c}\text { Número } \\
\text { de } \\
\text { máquinas }\end{array}$ & $\begin{array}{c}\text { Localização } \\
\text { (PO) }\end{array}$ & $\begin{array}{c}\text { Pontuação } \\
\text { atribuída } \\
\text { (consumo/h) } \\
\text { (1) }\end{array}$ & $\begin{array}{c}\text { Horas } \\
\text { trabalho } \\
\text { por } \\
\text { máquina }\end{array}$ & $\begin{array}{c}\text { Horas } \\
\text { trabalho } \\
\text { totais } \\
(2)\end{array}$ & $\begin{array}{c}\text { Pontuação } \\
\text { total } \\
\text { atribuída } \\
(3=1 \times 2) \\
\end{array}$ \\
\hline Chiron FZ c/ tanque & 26 & P01 & 1 & 5.496 & 142.885 & 142.885 \\
\hline 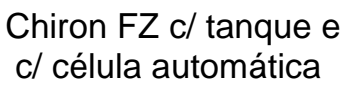 & 3 & PO2 & 1 & 4.705 & 14.115 & 14.115 \\
\hline Chiron FZ s/ tanque & 14 & P01 & 0,9 & 5.496 & 76.938 & 69.244 \\
\hline $\begin{array}{l}\text { Chiron FZ s/ tanque } \\
\text { c/ automação }\end{array}$ & 2 & PO4 & 0,9 & 6.786 & 13.573 & 12.216 \\
\hline Chiron DZ & 4 & $\mathrm{PO} 3$ & 1,5 & 5.236 & 20.945 & 31.418 \\
\hline Total & 49 & - & - & 27.719 & 268.456 & 269.878 \\
\hline
\end{tabular}

Nota. Fonte: Dados da pesquisa.

Com base nos critérios descritos na Tabela 3 para o direcionamento de cada uma das contas aos POs, a Tabela 4 mostra o resultado do foto-índice de cada um dos POs (FIPOs). O custo total de cada conta para cada PO foi dividido pelas horas planejadas. O FIPO é a soma desses valores horários para cada PO. 
Método de Custeio Tradicional versus Método da Unidade de Esforço de Produção (UEP): um estudo de caso em uma indústria multiprodutora do segmento metalmecânico

Maíra Vieira da Silva, Ricardo Gonçalves de Faria Corrêa

Tabela 4

Foto-índice dos Postos Operativos (FIPOs)

\begin{tabular}{|c|c|c|c|c|c|c|c|c|}
\hline \multirow{2}{*}{$\begin{array}{l}\text { Conta } \\
\text { Depreciação }\end{array}$} & \multicolumn{2}{|c|}{ P01 } & \multirow{2}{*}{$\begin{array}{c}\mathrm{PO2} \\
\mathrm{R} \$\end{array}$} & \multicolumn{2}{|r|}{ PO3 } & \multicolumn{3}{|c|}{$\mathrm{PO4}$} \\
\hline & $\mathrm{R} \$$ & 4,91 & & 4,69 & $\mathrm{R} \$$ & 18,61 & $\mathrm{R} \$$ & 11,16 \\
\hline Manutenção (materiais) & $\mathrm{R} \$$ & 2,33 & $\mathrm{R} \$$ & 5,12 & $\mathrm{R} \$$ & 6,29 & $\mathrm{R} \$$ & 1,28 \\
\hline Manutenção (serviços) & $\mathrm{R} \$$ & 0,80 & $\mathrm{R} \$$ & 1,06 & $\mathrm{R} \$$ & 1,04 & $\mathrm{R} \$$ & 0,61 \\
\hline Manutenção (horas) & $\mathrm{R} \$$ & 1,89 & $\mathrm{R} \$$ & 2,97 & $\mathrm{R} \$$ & 2,50 & $\mathrm{R} \$$ & 1,13 \\
\hline Energia Elétrica & $\mathrm{R} \$$ & 3,37 & $\mathrm{R} \$$ & 3,49 & $\mathrm{R} \$$ & 5,23 & $\mathrm{R} \$$ & 3,14 \\
\hline FIPO (R\$/h) & $\mathbf{R} \$$ & 13,30 & $\mathbf{R} \$$ & 17,32 & $\mathbf{R} \$$ & 33,67 & $\mathbf{R} \$$ & 17,32 \\
\hline
\end{tabular}

Nota. Fonte: Dados da pesquisa.

\subsubsection{Determinação do Foto-Custo Base}

Na etapa de cálculo do foto-custo base, através do produto base, definiram-se os produtos que seriam abordados na análise final deste estudo. A Tabela 5 mostra os tempos de passagem de cada produto pelos POs.

Tabela 5

Tempos de passagem dos produtos pelos POs (em h/uni.)

\begin{tabular}{lccccc}
\hline Produto & PO1 & PO2 & PO3 & PO4 & Tempo total \\
\hline Hobby-C-4 & 0,018 & - & - & - & 0,018 \\
Hobby-C-5 & 0,019 & - & - & - & 0,019 \\
Hobby-C-6 & 0,017 & - & - & - & 0,017 \\
Hobby-C-7 & 0,019 & - & - & - & 0,019 \\
Hobby-D13 & 0,035 & - & - & - & 0,035 \\
Inter-D-12 & - & 0,017 & - & - & 0,017 \\
Inter-E-14 & 0,027 & - & - & - & 0,027 \\
InterE-15 & 0,032 & - & - & - & 0,032 \\
Inter-F-11 & 0,021 & - & - & - & 0,021 \\
Profi-A-8 & 0,066 & - & - & - & 0,066 \\
Profi-A-9 & 0,055 & - & - & - & 0,055 \\
Profi-B-1 & 0,047 & - & 0,017 & - & 0,063 \\
Profi-B-2 & 0,054 & - & 0,018 & - & 0,072 \\
Profi-B-3 & - & - & 0,018 & 0,017 & 0,035 \\
Profi-F-10 & 0,046 & - & - & - & 0,046 \\
\hline
\end{tabular}

Nota. Fonte: Dados da pesquisa. 
Método de Custeio Tradicional versus Método da Unidade de Esforço de Produção (UEP): um estudo de caso em uma indústria multiprodutora do segmento metalmecânico Maíra Vieira da Silva, Ricardo Gonçalves de Faria Corrêa

Antunes Junior (1988) e Bornia (2010) relatam que o produto base deve ser definido através do produto que percorra em seu roteiro de produção todos os POs, pois ele teria a missão de representar os produtos da empresa no que diz respeito à utilização da estrutura produtiva. Relatam ainda que caso nenhum dos produtos passe por todos os POs, deve-se elencar um produto base fictício que assim faça. No entanto, em contribuição mais recente ao método da UEP, Moterle, Wernke e Zanin (2020) comprovam matematicamente, através de seu estudo de caso, que a escolha do produto base não exerce diferenciação no custo final unitário obtido pelo método da UEP. Dessa forma, tem-se então o conhecimento que, independentemente do produto a ser definido como produto-base o resultado obtido é o mesmo. Assim, o produto escolhido como produto base é o "Profi-B-1".

Através dos tempos de passagem do produto base (Profi-B-1) pelos POs, considerando os FIPOs encontrados na segunda etapa de aplicação do método, tem-se então o foto-custo base de $R \$ 1,18(R \$ 13,30 \times 0,047+R \$ 33,67 \times 0,017)$. A partir desse momento sabe-se que este é o custo de 1 UEP.

Quanto à escolha da amostragem de produtos para serem abordados na análise final, a mesma contempla um total de 15 produtos distintos, a fim de possuir uma amostra abrangente a todas as famílias de produtos fabricados na unidade de negócio 1 , bem como contemplando dentro de cada uma das famílias aqueles produtos que possuem maior representatividade em relação ao volume de produção. Os 15 produtos selecionados entre os 76 que são fabricados representam um total de aproximadamente 50\% do volume de produção do ano de 2019 .

\subsubsection{Cálculo dos Potenciais Produtivos}

À medida que se tem conhecimento dos FIPOs e do custo da unidade de UEP, é calculado o potencial produtivo de cada um dos POs em UEP, para então tratar apenas da unidade padrão: UEP. A Tabela 6 demonstra o potencial produtivo encontrado em cada um dos POs. 
Método de Custeio Tradicional versus Método da Unidade de Esforço de Produção (UEP): um estudo de caso em uma indústria multiprodutora do segmento metalmecânico

Maíra Vieira da Silva, Ricardo Gonçalves de Faria Corrêa

Tabela 6

Potenciais Produtivos dos POs

\begin{tabular}{|c|c|c|c|c|c|c|c|}
\hline POs & \multicolumn{2}{|c|}{ P01 } & \multicolumn{2}{|c|}{ PO2 } & \multicolumn{2}{|c|}{ PO3 } & PO4 \\
\hline FIPO (R\$/h) & $\mathrm{R} \$$ & 13,30 & $\mathrm{R} \$$ & 17,32 & $\mathrm{R} \$$ & 33,67 & $\mathrm{R} \$ \mathbf{1 7 , 3 2}$ \\
\hline Custo da UEP (R\$) & $\mathrm{R} \$$ & 1,18 & $\mathrm{R} \$$ & 1,18 & $\mathrm{R} \$$ & 1,18 & $\mathrm{R} \$ \quad 1,18$ \\
\hline Potenciais Procutivos (UEP/h) & \multicolumn{2}{|c|}{11,30} & \multicolumn{2}{|c|}{14,71} & \multicolumn{2}{|c|}{28,60} & 14,71 \\
\hline
\end{tabular}

Nota. Fonte: Dados da pesquisa.

Conforme a Tabela 6, o potencial produtivo é dado em UEP/h, a fim de indicar a quantidade de esforço que o PO exerce em uma hora de trabalho. Verifica-se que o potencial de esforço está intimamente ligado com o custo atrelado ao $\mathrm{PO}$, que por sua vez tem relação com o que o PO demanda em seu funcionamento habitual.

\subsubsection{Determinação dos Equivalentes dos Produtos em UEP}

A última fase de aplicação é dada com a determinação do quanto cada um dos produtos equivale em UEP, para que então se torne possível o custeio da produção pela UEP. Isso é obtido através dos tempos de roteiro dos produtos pelos POs, e do quanto cada um dos POs possui de potencial produtivo (UEP/h). A Tabela 7 mostra esse cálculo sintetizado para cada um dos produtos. 
Método de Custeio Tradicional versus Método da Unidade de Esforço de Produção (UEP): um estudo de caso em uma indústria multiprodutora do segmento metalmecânico

Maíra Vieira da Silva, Ricardo Gonçalves de Faria Corrêa

Tabela 7

Equivalente dos produtos em UEP

\begin{tabular}{lccccc}
\hline & & & & & \\
Produto & PO1 & PO2 & PO3 & PO4 & $\begin{array}{c}\text { Total } \\
\text { Equivalente } \\
\text { em UEP }\end{array}$ \\
\hline Hobby-C-4 & 0,20 & - & - & - & 0,20 \\
Hobby-C-5 & 0,21 & - & - & - & 0,21 \\
Hobby-C-6 & 0,19 & - & - & - & 0,19 \\
Hobby-C-7 & 0,21 & - & - & - & 0,21 \\
Hobby-D-13 & 0,40 & - & - & - & 0,40 \\
Inter-D-12 & - & 0,25 & - & - & 0,25 \\
Inter-E-14 & 0,30 & - & - & - & 0,30 \\
Inter-E-15 & 0,36 & - & - & - & 0,36 \\
Inter-F-11 & 0,24 & - & - & - & 0,24 \\
Profi-A-8 & 0,74 & - & - & - & 0,74 \\
Profi-A-9 & 0,62 & - & - & - & 0,62 \\
Profi-B-1 & 0,53 & - & 0,47 & - & 1,00 \\
Profi-B-2 & 0,60 & - & 0,52 & - & 1,12 \\
Profi-B-3 & - & - & 0,52 & 0,25 & 0,76 \\
Profi-F-10 & 0,52 & - & - & - & 0,52 \\
\hline
\end{tabular}

Nota. Fonte: Dados da pesquisa.

Por fim, são apresentadas as relações de esforços demandados por cada um dos produtos, que variam de acordo com o tempo de passagem desses produtos por diferentes pontos do processo de produção e pela capacidade de esforço atrelada a cada um destes pontos (POs).

\subsection{Custeio Final pelo Método da UEP e Método dos Centros de Custos}

Conforme mencionado na etapa final de aplicação da UEP, uma vez que é conhecido o custo da unidade (UEP) e o quanto cada um dos produtos equivale em UEP, o cálculo de custo final se torna viável e simples. A Tabela 8 exibe o custo de cada um dos produtos no processo de usinagem, pelo método da UEP e pelo método dos CC, considerando a parcela de $43,70 \%$ das contas abordadas. 
Método de Custeio Tradicional versus Método da Unidade de Esforço de Produção (UEP): um estudo de caso em uma indústria multiprodutora do segmento metalmecânico Maíra Vieira da Silva, Ricardo Gonçalves de Faria Corrêa

Tabela 8

Custo dos produtos pelos métodos da UEP e CC

\begin{tabular}{|c|c|c|c|c|c|}
\hline Produto & $\begin{array}{l}\text { Equivalente } \\
\text { em UEP }\end{array}$ & $\begin{array}{c}\text { Custo final } \\
\text { Método UEP (a) }\end{array}$ & $\begin{array}{l}\text { Custo final } \\
\text { Método dos } \\
\text { CC's (b) }\end{array}$ & Delta (c=a-b) & $\begin{array}{c}\text { Delta em \% } \\
(=\mathrm{c} / \mathrm{b})\end{array}$ \\
\hline Hobby-C-4 & 0,2 & 0,23 & 0,27 & 0,04 & $-13,10 \%$ \\
\hline Hobby-C-5 & 0,21 & 0,25 & 0,29 & 0,04 & $-13,10 \%$ \\
\hline Hobby-C-6 & 0,19 & 0,23 & 0,26 & 0,03 & $-13,10 \%$ \\
\hline Hobby-C-7 & 0,21 & 0,25 & 0,29 & 0,04 & $-13,10 \%$ \\
\hline Hobby-D-13 & 0,4 & 0,47 & 0,54 & 0,07 & $-13,10 \%$ \\
\hline Inter-D-12 & 0,25 & 0,3 & 0,26 & 0,03 & $-13,20 \%$ \\
\hline Inter-E-14 & 0,3 & 0,36 & 0,41 & 0,05 & $-13,10 \%$ \\
\hline Inter-E-15 & 0,36 & 0,42 & 0,49 & 0,06 & $-13,10 \%$ \\
\hline Inter-F-11 & 0,24 & 0,28 & 0,32 & 0,04 & $-13,10 \%$ \\
\hline Profi-A-8 & 0,74 & 0,88 & 1,01 & 0,13 & $-13,10 \%$ \\
\hline Profi-A-9 & 0,62 & 0,72 & 0,83 & 0,11 & $-13,10 \%$ \\
\hline Profi-B-1 & 1 & 1,18 & 0,97 & 0,21 & $21,64 \%$ \\
\hline Profi-B-2 & 1,12 & 1,32 & 1,1 & 0,23 & $20,56 \%$ \\
\hline Profi-B-3 & 0,76 & 0,9 & 0,53 & 0,37 & $68,92 \%$ \\
\hline Profi-F-10 & 0,52 & 0,61 & 0,7 & 0,09 & $-13,10 \%$ \\
\hline
\end{tabular}

Nota. Fonte: Dados da pesquisa.

O valor apresentado na coluna (a) trazendo o custo final dos produtos pelo método da UEP tem seu resultado apurado através do cálculo de Equivalente em UEP $x R \$ 1,18$ (custo da UEP). Vale ressaltar que a utilização dessa metodologia para o custeio real dos produtos mês a mês se apresenta de maneira bastante simples, dado que uma vez que se conhece o quanto cada produto demanda de esforço, o que se altera mensalmente é o valor econômico da UEP.

O valor apresentado na coluna (b) trazendo o custo final dos produtos pelo método dos $\mathrm{CC}$ tem seu resultado apurado através do cálculo do tempo total do produto no processo (conforme dados da Tabela 5) x $\mathrm{R} \$ 15,30$ (tarifa horária do CC). A tarifa de $\mathrm{R} \$ 15,30$ é obtida ao se considerar também o percentual de $43,70 \%$ das contas que 
Método de Custeio Tradicional versus Método da Unidade de Esforço de Produção (UEP): um estudo de caso em uma indústria multiprodutora do segmento metalmecânico

Maíra Vieira da Silva, Ricardo Gonçalves de Faria Corrêa

foram contempladas na aplicação do método da UEP. As variações expostas na Tabela 8 tem espaço para discussão na seção seguinte.

\section{ANÁLISE DOS RESULTADOS}

Comparando os métodos, observa-se que em todos os produtos são encontradas diferenças no valor custeado, conforme o método utilizado. As diferenças são de natureza exclusiva da sistemática de cada método de custeio. Assim, faz-se necessária a reflexão e análise para entender e contrastar os custos dos produtos obtidos pelas duas metodologias.

A Figura 3 ilustra as diferenças de custeio encontradas nos produtos abordados por este estudo. Esses dados também podem ser observados ao final da seção 4.4 na Tabela 8.

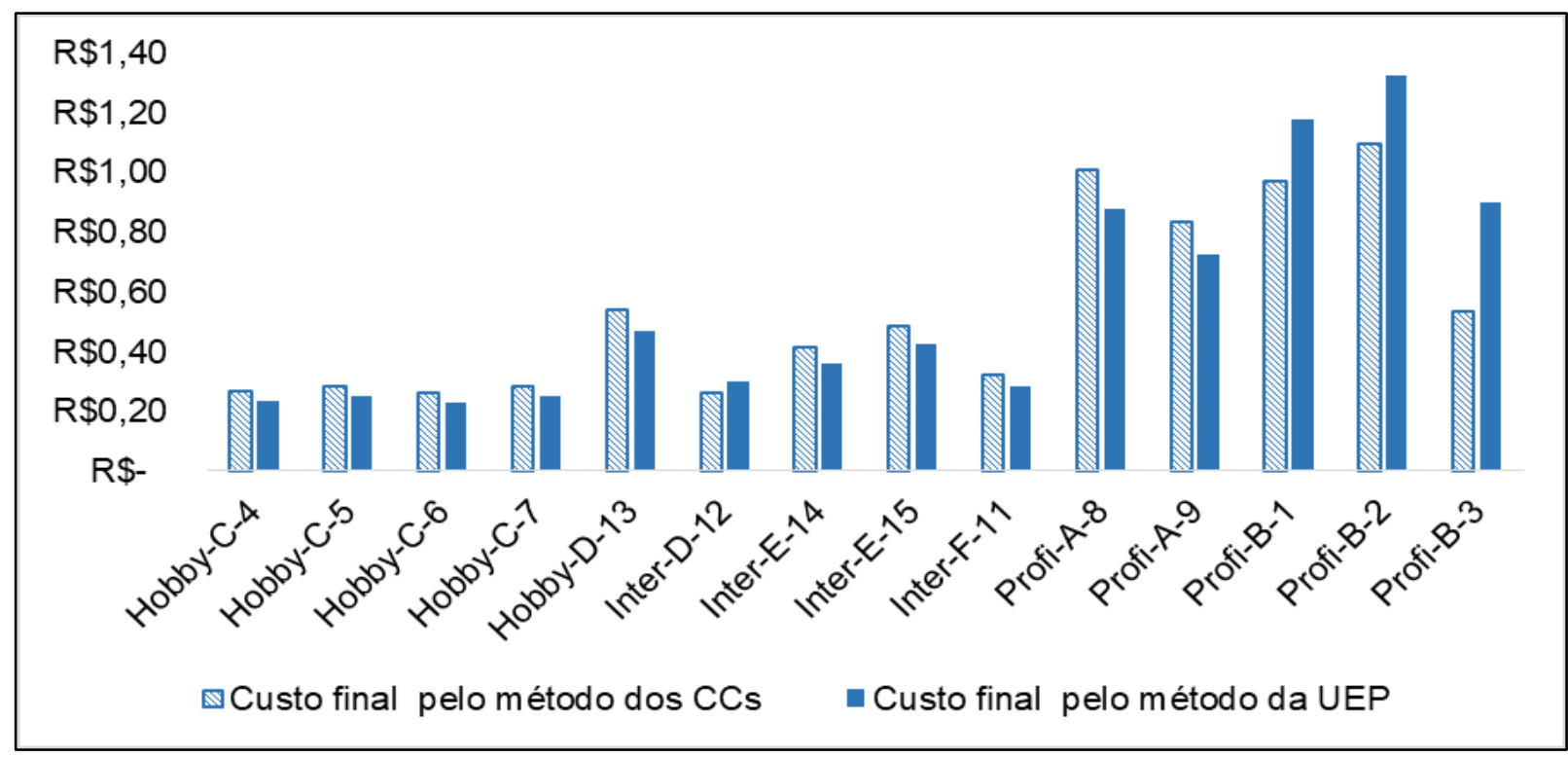

Figura 3. Custo final dos produtos pelos métodos da UEP e CC

Fonte: Dados da pesquisa.

Nota-se que os quatro primeiros produtos apresentados na Figura 3 (linha Hobby), pertencentes à família de produtos fundidos sob pressão, apesar de possuírem 
Método de Custeio Tradicional versus Método da Unidade de Esforço de Produção (UEP): um estudo de caso em uma indústria multiprodutora do segmento metalmecânico Maíra Vieira da Silva, Ricardo Gonçalves de Faria Corrêa

os menores custos pelo método dos CC, pelo método da UEP seriam custeados por menos ainda. Verifica-se que esse resultado apresenta coerência, visto que esses produtos possuem em seu roteiro de produção somente o PO1, que pela sistemática da UEP apresenta o menor esforço (UEP/h) entre os POs.

Já o produto Hobby-D-13 apresenta um custo elevado se comparado aos demais produtos da mesma classe. Isso se deve ao fato desse item possuir um item a mais em sua composição (bucha) e demandar mais tempo de usinagem no PO1. Ainda assim, esse produto - bem como os demais da classe Hobby - é penalizado em seu custeio final pelo método dos CC, quando comparado com o da UEP.

De acordo com o método da UEP, o produto Inter-D-12 deveria ter seu custo acrescido quando comparado com o custo do método dos CCs. Isso se valida em razão do produto ter seu roteiro concentrado exclusivamente no PO2, que possui um custo horário mais elevado do que a tarifa média utilizada no método atual.

O custo dos produtos Hobby-D-13 e Inter-D-12 destoam em relação às suas famílias. Isso ocorre pois o produto Hobby-D-13 ao ser usinado no P01 possui um tempo de roteiro maior se comparado ao produto Inter-D-12 que é usinado no PO2, posto esse mais rápido por contar com célula automática para alimentação. Essa relação serve como um exemplo de ganho do PO2 frente ao PO1, visto que o PO2 apesar de desprender um esforço maior do que o PO1 apresenta uma velocidade maior, mostrando-se como uma alternativa mais interessante nesse caso.

Analisando os produtos de classificação profissional, verifica-se que os produtos da família B são os que apresentam as variações mais agressivas em relação ao custeio final por ambos os métodos. São encontradas variações de 20,56\%, 21,64\% e até mesmo 68,92\%. Tamanha variação encontrada é reflexo da aplicação de um método que separa os recursos de acordo com o esforço para a fabricação dos produtos. Dessa maneira, o resultado de custeio apresentado pelo método da UEP para esses produtos possui coerência, visto que na separação dos POs, o PO 3, posto no qual a maioria das fases de usinagem desses produtos é realizada, apresenta uma quantidade de esforço maior. Verifica-se, assim, que pela metodologia utilizada 
Método de Custeio Tradicional versus Método da Unidade de Esforço de Produção (UEP): um estudo de caso em uma indústria multiprodutora do segmento metalmecânico Maíra Vieira da Silva, Ricardo Gonçalves de Faria Corrêa

atualmente pela empresa esses produtos são fortemente subsidiados pelos produtos das famílias com um nível menor de complexidade.

Referente aos demais produtos da linha Profi: A-8, A-9 e F-10, a utilização da UEP demonstra que esses produtos devem custar menos do que o que consta atualmente, pelo mesmo motivo citado anteriormente em outros casos, pois esses produtos apesar de profissionais possuem suas fases de usinagem concentradas no PO1. A diferenciação dos produtos profissionais da família $B$, frente aos produtos também profissionais das famílias $A$ e $F$, se dá pelos mesmos possuírem uma tecnologia associada diferenciada e de complexidade superior.

A aplicação do método da UEP oferece um campo de reflexão diante da realidade na qual a empresa opera e se encontra inserida. Atualmente os produtos Hobby C-4, C-5, C-6 e C-7, que representam cerca de $16 \%$ do volume total de produção da unidade de negócio, enfrentam disputa por mercado com concorrentes chineses, possuindo projetos de redução de custos direcionados especialmente para a redução de custo unitário desses modelos. Entretanto, essas ações são relatadas com alto índice de dificuldade para efetivação, por tratar-se de produtos que já possuem um custo baixo perante os demais. Além disso, verificam-se relatos de que a complexidade e dificuldade encontrada pelas equipes e especialistas para visualização de qual ação realiza impacto positivo na melhoria do custo, é alta.

Através da aplicação do método da UEP torna-se claro que esses produtos devem possuir um custo unitário menor, se custeados de maneira coerente em relação ao seu processo de transformação. Já ao considerar os produtos Profi (B-1, B-2 e B-3), que atualmente têm seu custo sendo subsidiado, a variação positiva no custo não necessariamente é um agravante, dado que esse produto está inserido em um mercado que possui maior capacidade para receber essa variação, ao contrário do primeiro grupo.

Evidencia-se, assim, através da aplicação da UEP, que a metodologia de custeio a ser utilizada impacta em um número maior de questões do que normalmente costuma-se enxergar, o que converge com a orientação de Bornia (2010). O 
Método de Custeio Tradicional versus Método da Unidade de Esforço de Produção (UEP): um estudo de caso em uma indústria multiprodutora do segmento metalmecânico Maíra Vieira da Silva, Ricardo Gonçalves de Faria Corrêa

direcionamento equivocado do custo do processo ao produto, de acordo com o que ele realmente demanda ao ser produzido, tem o poder de distorcer o foco das ações que realmente se fazem necessárias, podendo direcionar equipes a projetos sem retorno ou até mesmo a perdas de posicionamento no mercado. Essa conclusão tangencia a descoberta de Wernke et al. (2021) que identificaram que quanto mais precisa e individualizada a aplicação da UEP, mais precisa é a atribuição de perdas, como ociosidade.

Por fim, vale ressaltar, que ao encontrar os POs que possuem um nível maior de esforço atrelado e consequentemente de custo maior, a ação não deve ser simplesmente evitar que os produtos sejam ali fabricados, mas sim de direcionar corretamente ações para a melhoria da performance nesses postos, alcançando assim melhores resultados. Essa proposição coincide com os achados de Campagnolo et al. (2009).

\section{CONCLUSÃO}

A partir da aplicação do método da UEP tornou-se possível enxergar os benefícios de uma metodologia de custeio para além do custeio dos produtos. Assim, atendeu-se o objetivo avaliar e comparar os resultados da aplicação do método da UEP com o método dos CC. O que permitiu fazer reflexões acerca da competitividade dos produtos, de acordo com seu custeio atual e com a competitividade e cenário de mercado para qual cada família de produtos se destina.

Além disso, verificaram-se pontos como a importância de um custeio acurado até mesmo para o direcionamento de ações de redução de custos melhor sucedidas.

Pode-se constatar que o método tradicional dos centros de custos não proporciona um atendimento satisfatório devido à sua sistemática generalista. No contexto de uma empresa multiprodutora e com heterogeneidade no processo produtivo, o método de custeio da UEP possui melhor capacidade de atribuir os custos aos produtos, considerando as suas especificidades. 
Método de Custeio Tradicional versus Método da Unidade de Esforço de Produção (UEP): um estudo de caso em uma indústria multiprodutora do segmento metalmecânico Maíra Vieira da Silva, Ricardo Gonçalves de Faria Corrêa

Para chegar ao final deste estudo com essa constatação, as etapas iniciais foram de grande valia. O entendimento e apropriação em relação ao método da UEP, através de pesquisas e das visitas de benchmarking, garantiram a preparação necessária para enfrentar o desafio da aplicação do método.

Como fruto da maneira com que as etapas iniciais mencionadas foram abordadas, pode-se garantir a precisão da aplicação do método da UEP. A etapa de coleta e depuração dos dados mostrou-se como a mais desafiadora, pois primou-se a observância ao princípio das estratificações, garantindo que todas as contas consideradas possuíam dados validados e confiáveis.

Como limitações destacam-se a aplicação parcial do método da UEP, sem os indicadores de desempenho do processo. Isso se deve a outra limitação relacionada à aplicação parcial do método, que ocorreu como projeto piloto. Tais limitações não diminuem os resultados, porém não revelam todo potencial do método da UEP e dificultam a percepção do tomador de decisão para avaliar o método como superior ao CC. Assim, sugere-se para a condução de pesquisas futuras que os demais itens de custos sejam inseridos na sistemática de aplicação do método, para que, assim, possa se ter uma visão integral quanto ao custeio dos produtos.

\section{REFERÊNCIAS}

Allora, F., \& Allora, V. (1995). UP': unidade de medida da produção para custos e controles gerenciais das fabricações. São Paulo, Pioneira.

Antunes Júnior, J. A. V. (1988). Fundamentação do método das unidades de esforço de produção. (Dissertação de Mestrado). Universidade Federal de Santa Catarina, UFSC, Florianópolis, Santa Catarina.

Bornia, A. C. (2010). Análise gerencial de custos: aplicação em empresas modernas. São Paulo, Atlas.

Campagnolo, R. R., Souza, J. S., \& Kliemann Neto, F. J. (2009, setembro). Seria mesmo o Time-Driven ABC (TDABC) um método de custeio inovativo? Uma análise comparativa entre o TDABC e o método da unidade de esforço de produção (UEP). Anais do Congreso Internacional de Costos y Gestion, Patagonia, Argentina, 11. 
Método de Custeio Tradicional versus Método da Unidade de Esforço de Produção (UEP): um estudo de caso em uma indústria multiprodutora do segmento metalmecânico Maíra Vieira da Silva, Ricardo Gonçalves de Faria Corrêa

Crepaldi, S. A., \& Crepaldi, G. S. (2017). Contabilidade gerencial: teoria e prática. São Paulo, Atlas.

Creswell, J. W. (2010). Projeto de pesquisa: Métodos qualitativo, quantitativo e misto. Porto Alegre, Artmed.

Dresch, A., Lacerda, D. P., \& Miguel, P. A. C. (2015). Uma análise distintiva entre o Estudo de Caso, a Pesquisa-Ação e a Design Science Research. Revista Brasileira de Gestão de Negócios, 17 (56), 1116-1133.

Fadanelli, V. G. (2007). A utilização do método da Unidade de Esforço de Produção como modelo de gestão de custos - O caso de uma empresa do ramo metalúrgico. (Dissertação de Mestrado). Universidade Federal do Rio Grande do Sul, UFRGS, Porto Alegre, Rio Grande do Sul.

Fernandes, J. S. (2003). Sistematização de uma abordagem da medição de uma produção diversificada e seus desempenhos num ambiente industrial pelo método das unidades de esforço de produção - UEPs. (Dissertação de Mestrado). Universidade Federal do Rio Grande do Sul, UFRGS, Porto Alegre, Rio Grande do Sul.

Gil, A. C. (2018). Como elaborar projetos de pesquisa. São Paulo, Atlas.

Guimarães Filho, L. P., Bristot, V. M., Marques, L. R., Feil, N. F., \& Colombo, T. C. (2016). Aplicação do método da UEP na determinação dos custos de uma empresa de revestimentos cerâmicos. ABCustos, 11(3), 28-61.

Kliemann Neto, F. J. (1994, novembro). Gerenciamento e controle da produção pelo método de esforço de produção. Anais do Congresso brasileiro de gestão estratégica de custos, São Leopoldo, RS, Brasil, 1.

Kraemer, T. H. (1995). Discussão de um sistema de custeio adaptado às exigências da nova competição global. (Dissertação de Mestrado). Universidade Federal do Rio Grande do Sul, UFRGS, Porto Alegre, Rio Grande do Sul.

Lembeck, M., \& Wernke, R. (2019). Método UEP aplicado à pequena empresa fabril: uma relação custo/benefício favorável. ABCustos, 14 (3), 26-55.

Marconi, M. A., \& Lakatos, E. M. (2017). Fundamentos da metodologia científica. São Paulo, Atlas. 
Método de Custeio Tradicional versus Método da Unidade de Esforço de Produção (UEP): um estudo de caso em uma indústria multiprodutora do segmento metalmecânico Maíra Vieira da Silva, Ricardo Gonçalves de Faria Corrêa

Martins, H. H. T. S. (2004) Metodologia qualitativa de pesquisa. Educação e Pesquisa, 30 (2), 289-300.

Moterle, S., Wernke, R., \& Zanin, A. (2020). Influência da escolha do produto-base do método unidades de esforço de produção (UEP) no custo unitário de transformação. Exacta Engenharia de Produção. 18 (4). 758-777.

Muller, J. C. (1996). A evolução dos sistemas de manufatura e a necessidade de mudança nos sistemas de controle e custeio. (Dissertação de Mestrado). Universidade Federal do Estado do Rio Grande do Sul, UFRGS, Porto Alegre, Rio Grande do Sul.

Oliveira, S. E., \& Allora, V. (2010). Gestão de custos: metodologia para a melhoria da performance empresarial. Curitiba, Juruá.

Padoveze, C. L. (2010). Contabilidade gerencial: um enfoque em sistema de informação contábil. São Paulo, Atlas.

Pasa, R., Nascimento, S., \& Correio, I. B. D. (2017). Gestão estratégica de custos: análise da produção científica na ABCustos no período de 2006 a 2015. ABCustos, 12 (2), 82-110.

Silva, A. C. R., Cruz, V. L., Santos, R. R., \& Leone, R. J. G. (2018). Análise da gestão estratégica de custos e métodos de custeio tradicionais em uma indústria têxtil paraibana. ABCustos, 13 (1), 58-76.

Souza, A. \& Clemente, A. (2011). Gestão de Custos: aplicações operacionais e estratégicas exercícios resolvidos e propostos com utilização do EXCEL. São Paulo, Atlas.

Souza, M. A. \& Diehl, C. A. (2009). Gestão de Custos: uma abordagem integrada entre contabilidade, engenharia e administração. São Paulo, Atlas.

Valentim, T. L. S. (2018). Avaliação de contribuições teóricas ao método das Unidades de Esforço de Produção (UEPs). ABCustos, 13 (2), 01-26.

Villarmois, O. de La., Levant, Y. (2011). From adoption to use of a management control tool: case study evidence of a costing method. Journal of applied accounting research, v. 12, n. 3, p. 234-259.

Wernke, R., Junges, I., \& Zanin, A. (2019). Mensuração da ociosidade fabril pelos métodos ABC, TDABC e UEP. Revista Contemporânea de Contabilidade, 16 (38), 185-206. 
Método de Custeio Tradicional versus Método da Unidade de Esforço de Produção (UEP): um estudo de caso em uma indústria multiprodutora do segmento metalmecânico

Maíra Vieira da Silva, Ricardo Gonçalves de Faria Corrêa

Wernke, R., Rufatto, I., \& Lembeck, M. (2021). UEP específica por Linha de Produção ou UEP única para a Fábrica toda? ABCustos, v. 16, n. 1.

Zanin, A., Bilibio, A., Pacassa, F., \& Cambruzzi, C. (2019) O método de custeio UEP como fonte geradora de informações gerenciais: estudo multicasos. ABCustos, 14 (3), 143-164.

Data de Submissão: 11/11/2020

Data de Aceite: 05/08/2021 